\title{
Aplicação do Método Kinesio Taping e seus efeitos na redução da dor e no ganho da flexibilidade da coluna lombar em funcionários que trabalham sentados
}

\author{
Application of the Kinesio Taping Method and its effects on pain reduction and gain of \\ lumbar spine flexibility in sitting staff
}

Aplicación del Método Kinesio Taping y sus efectos en la reducción del dolor y en la ganancia de la flexibilidad de la columna lumbar en funcionarios que trabajan sentados

Heloene de Carvalho Lima ${ }^{1 *}$, Karla Rakel Gonçalves Luz², Marcio Marinho Magalhães ${ }^{1}$, Daniel Miranda da Silva ${ }^{1}$, Michele Araújo da Silva Reis ${ }^{1}$, Erica Karoline Carvalho Costa Rodrigues ${ }^{1}$, Jaynara Wanderley de Morais ${ }^{1}$, Felipe Batista Santo ${ }^{3}$, Fernando Ribeiro Castro ${ }^{4}$.

\section{RESUMO}

Objetivo: Avaliar se o método Kinesio Taping reduz a dor lombar associada à postura de trabalho sentado. Verificar se a utilização da fita melhora a flexibilidade lombar. Avaliar a intensidade da dor lombar através da escala de dor e utilizar o método Kinesio Taping em funcionários com dor lombar que trabalham sentados para avaliar uma possível melhora. Métodos: Tratou-se de um ensaio clínico, randomizado e controlado, de caráter quantitativo, onde se utilizou a Escala Visual Analógica (EVA), inspeção e palpação, medição mãoterra e a Kinesio Taping. Participaram do estudo 50 funcionários, sendo 25 do grupo Kinesio Taping e 25 do grupo controle. Resultados: Os funcionários avaliados que trabalham na postura sentada são mais acometidos por dor lombar de grau moderada de acordo com a escala EVA. Com a aplicação da Kinesio Taping pode-se observar uma redução do quadro álgico na região lombar sendo estatisticamente significativo com $p=0,05$, que foi avaliado pela mesma escala e aumento da flexibilidade da coluna lombar, sendo extremamente significativo com $\mathrm{p}=0,000$, na qual foi avaliada pela medição mão-terra. Conclusão: $O$ método Kinesio Taping foi eficaz na redução do quadro álgico e no ganho de flexibilidade lombar dos funcionários que trabalham na postura sentada.

Palavras-chave: Dor lombar, Flexibilidade, Kinesio Taping, Fisioterapia.

\begin{abstract}
Objective: To evaluate if the Kinesio Taping method reduces the low back pain associated with sitting posture. Check if tape use improves lumbar flexibility. Evaluate the intensity of low back pain through the pain scale and use the Kinesio Taping method on employees with low back pain who work sitting to evaluate a possible improvement. Methods: This was a randomized, controlled, quantitative clinical trial using the Analogic visual scale (EVA), inspection and palpation, hand-to-ground measurement, and Kinesio Taping. Fifty employees participated, of which 25 were from the Kinesio Taping group and 25 from the control group. Results: The evaluated workers who work in the seated posture are more affected by moderate-grade low back pain according to the EVA scale. With the application of Kinesio Taping, a reduction of the pain in the lumbar region was observed, being statistically significant with $p=0.05$, which was evaluated by the same scale and increase of lumbar spine flexibility, being extremely significant with $p=0.000$, in which it was evaluated by hand-toground measurement. Conclusion: The Kinesio taping method was effective in reducing the pain and lumbar flexibility of sitting staff.
\end{abstract}

Keywords: Lumbar pain, Flexibility, Kinesio Taping, Physiotherapy.

${ }^{1}$ Centro Universitario de Ciências e Tecnologia do Maranhão - UNIFACEMA. Caxias - MA.

*E-mail: heloenecarvalhofisio@gmail.com

${ }^{2}$ Conselho Federal de Fisioterapia e Terapia Ocupacional. Teresina - PI.

${ }^{3}$ Faculdade Dom Bosco. São Luís - MA.

${ }^{4}$ Universidade Federal do Maranhão. São Luís - MA. 


\section{RESUMEN}

Objetivo: Evaluar si el método Kinesio Taping reduce el dolor lumbar asociado a la postura de trabajo sentado. Comprobar si el uso de la cinta mejora la flexibilidad lumbar. Evaluar la intensidad del dolor lumbar a través de la escala de dolor y utilizar el método Kinesio Taping en empleados con dolor lumbar que trabajan sentados para evaluar una posible mejora. Métodos: Se trató de un ensayo clínico, randomizado y controlado, de carácter cuantitativo, donde se utilizó la Escala visual analógica (EVA), inspección y palpación, medición mano-tierra y la Kinesio Taping. Participaron del estudio 50 empleados, siendo 25 del grupo Kinesio Taping y 25 del grupo control. Resultados: Los funcionarios evaluados que trabajan en la postura sentada son más acometidos por dolor lumbar de grado moderado de acuerdo con la escala EVA. Con la aplicación del Kinesio Taping se puede observar una reducción del cuadro álgico en la región lumbar siendo estadísticamente significativo con $p=0,05$, que fue evaluado por la misma escala y aumento de la flexibilidad de la columna lumbar, siendo extremadamente significativo con $p=0,000$, en la cual fue evaluada por la medición manotierra. Conclusión: El método Kinesio Taping fue eficaz en la reducción del cuadro álgico y en la ganancia de flexibilidad lumbar de los empleados que trabajan en la postura sentada.

Palabras clave: Dolor lumbar, Flexibilidad, Kinesio Taping, Fisioterapia.

\section{INTRODUÇÃO}

A dor lombar é um distúrbio musculoesquelético de característica multifatorial, que atinge ambos os sexos, é muito recorrente na população financeiramente ativa, levando temporária ou permanentemente à incapacidade laboral, a mesma tornou-se um grande problema de saúde pública em diversos países, não só pela sua alta prevalência e incidência, mas também pelo uso abundante dos serviços de saúde e pelo afastamento de trabalho, se destacando, entre as principais causas de invalidez (BARROS SS, et al., 2011; PATARO SMS e FERNANDES RCP, 2014).

São diversos os fatores associados à dor lombar, contudo sua origem está constantemente ligada à fadiga e à posturas inapropriadas e repetitivas, determinadas por atividades ocupacionais em que o trabalhador permanece em posições antiergonômicas por longos períodos de tempo, uma vez que a permanência de uma posição estática não faz parte do modelo anatômico e funcional do corpo humano (BARROS SS, et al., 2011).

Não há uma definição para padrões exatos de flexibilidade, mas sabe-se que sua diminuição e/ou ausência limita a mobilidade e pode exacerbar a probabilidade de lesão na articulação e nos músculos ali envolvidos. As estruturas que compõe a coluna vertebral estão diariamente sujeita a vícios posturais, em especial os músculos, que podem levar a um desequilíbrio e ao encurtamento, ocasionando uma alteração de postura e o surgimento de algias (LIMA JMR, 2016).

A posição sentada é a que mais causa desarmonia na postura corporal, visto que o homem moderno permanece, em média, um terço da sua vida nessa postura e não pratica atividade física, o que pode acarretar em alterações biomecânicas, como desequilíbrio muscular entre força extensora e flexora do tronco e redução da estabilidade e mobilidade do complexo lombo-pelve-quadril, responsáveis pelo desenvolvimento de dores na porção inferior da coluna (FREITAS KPN, et al., 2011).

A fisioterapia usufrui de diversos métodos para a redução da dor lombar, como a cinesioterapia, eletrotermofototerapia, terapias chinesas, mobilizações, dentre outros, e um novo método que vem sendo utilizado no Brasil e que pode ajudar na redução do quadro álgico desses pacientes é a Kinesio Taping (KT), que atua através da estimulação de mecanorreceptores diminuindo a pressão da pele sobre o tecido miofascial, alterando o fluxo sanguíneo, facilitando na redução do processo inflamatório, do quadro álgico, auxiliando na reabilitação do sistema musculoesquelético e fornecendo suporte simultâneo às articulações (REINEHR FB, et al., 2017; ALONSO AC, et al., 2015).

O método fornece resultados por intermédios biomecânicos e neurofisiológicos, no entanto o tratamento da dor e de patologias do sistema nervoso baseiam-se sobretudo nos efeitos neurofisiológicos, as aplicações fisiológicas abordam a correção de atividades muscular, melhora a flexibilidade, favorece a circulação sanguínea e linfática, diminui a dor pela supressão neurológica e pelo reposicionamento das articulações (DE OLIVEIRA LR e MEJIA DPM, 2012; VARGAS B, et al., 2014). 
A KT vem sendo usada há muito tempo como método de tratamento, mas somente há 20 anos se tornou uma abordagem fisioterapêutica, uma vez que possui vasta aplicabilidade e pode ser usada nas disfunções neurais, musculares, fasciais e articulares em todas as partes do corpo (THOMPSON D, 2010).

O principal propósito desse estudo é avaliar se o método Kinesio Taping reduz a dor e melhora a flexibilidade lombar associada à postura de trabalho de funcionários que trabalham sentados. E como objetivos específicos, avaliar a intensidade da dor lombar através da escala de dor e utilizar a Kinesio Taping em funcionários com dor lombar que trabalham sentados para avaliar uma possível melhora.

\section{METODOLOGIA}

Tratou-se de um ensaio clínico, randomizado e controlado, de caráter quantitativo, que foi executado na Faculdade de Ciências e Tecnologia do Maranhão-FACEMA. A amostra foi composta por 50 funcionários, que foram selecionados e distribuídos em dois grupos distintos, sendo um grupo controle (GC), composto por 25 funcionários, e um grupo KT (GKT), contendo 25 funcionários.

Foram inclusos na pesquisa apenas funcionários que trabalhavam sentados, que referiam dor lombar e que estavam de acordo a participar da mesma. E foram desconsiderados os funcionários que relataram ter alteração de sensibilidade, alergia a substância adesiva da KT, e aqueles que desistiram ou não compareceram aos procedimentos.

A coleta de dados foi realizada através de um questionário constituído por 12 questões, uma ficha de avaliação que foi aplicada em dois momentos e a aplicação da KT na região lombar.

A primeira etapa da ficha de avaliação abordou as informações gerais dos participantes, aferição da intensidade da dor lombar, que fora avaliada pela escala visual analógica (EVA) e teste da medição mãoterra, que avaliou a mobilidade da coluna lombar em flexão; e a $2^{\text {a }}$ após 4 dias de aplicação da KT com utilização da escala visual analógica e medição mão-terra.

A escala visual analógica (EVA) é utilizada para quantificar a intensidade da dor e é compreendida por uma linha horizontal, numerada de zero a dez, onde zero indica sem dor e dez a dor mais insuportável sentida pelo funcionário, sua numeração segue a pontuação ordinal, sendo possível classificar a dor em leve, moderada e intensa, para cada classe da sua mensuração há um desenho de duas carinhas que indica a expressão facial, visando facilitar a identificação da intensidade dolorosa sentida pelo participante (CARAVIELLO EZ, et al., 2016; MASCARENHAS CHM e SANTOS LS, 2011).

Existem diversos métodos que são utilizados para avaliar a mobilidade do tronco, porém, o teste de medição mão-terra, por ser uma avaliação quantitativa, se torna mais confiável, fornecendo dados mais fidedignos. O mesmo é utilizado para verificar a mobilidade da coluna em flexão, onde o funcionário com os joelhos estendidos deverá fletir seu tronco e tentar tocar o chão com a mão, caso este não consiga, será medido com uma régua, a distância do terceiro dedo ao chão (HOPPENFELD S, 2003).

Após o questionário e a ficha de avaliação, foi aplicado a KT, no GKT de acordo com a padronização do método proposto pelo criador Kenso Kase. Para a aplicação foi solicitado que o participante realizasse flexão de tronco, a KT foi posicionada na região de paravertebrais bilateralmente, paralelas aos processos espinhosos das vertebras lombares, em forma de "l", iniciando próximo a espinha ilíaca póstero-superior até nível de T12. A tensão que foi utilizada é caracterizada como muito leve, sendo de 10-15\% na zona terapêutica e $0 \%$ nas âncoras.

Antes do procedimento foi realizada a assepsia da região lombar com algodão e leite de magnésia para promover uma melhor aderência da fita e a cantoplastia da KT (retirada dos cantos) para evitar a soltura da mesma. Após a aplicação da KT o participante foi orientado a permanecer com a fita por um período de 4 dias, podendo realizar suas atividades normalmente.

Além da coleta de dados fez-se um levantamento bibliográfico nas bases de dados Biblioteca Virtual em Saúde (BVS), Biblioteca Nacional de Medicina dos Estados Unidos (PUBMED), Literatura Latino-Americana 
e do Caribe em Ciências da Saúde (LILACS), Physiotherapy Evidence Database (PEDro) e Scientific Electronic Library Online (SciELO), com os descritores: Dor lombar, Kinesio Taping e Fisioterapia, além de livros a fim de complementar as informações inerentes ao proposto tema.

Os dados foram organizados e tabulados utilizando o SPSS versão 23.0 e apresentados por meio de frequência simples e absoluta. Para verificar associação entre as variáveis foi utilizado o teste exato de qui quadrado $(p)$, considerando em todas as análises realizadas um nível de significância de $5 \%$.

Os funcionários da Faculdade de Ciências e Tecnologia do Maranhão - FACEMA envolvidos na pesquisa foram abordados, na qual foi explicado sobre o Termo de Consentimento Livre e Esclarecido (TCLE) respeitando os aspectos éticos que envolve os estudos dessa natureza, respaldada na resolução 466/2012 do Conselho Nacional de Saúde, que institui as normas de pesquisa em saúde. Após tomarem ciência e concordarem em participar de todas as etapas do estudo foi explanado sobre o projeto. Essa pesquisa foi aprovada pelo Comitê de Ética em pesquisa sob o número do parecer 2.492.585.

O estudo apresentava riscos mínimos, mas estavam relacionados com alergia ao material da fita adesiva, desistência do voluntário e constrangimento em responder as questões. Para minimizar os mesmos realizouse uma aplicação da KT sobre a pele do voluntário para verificar se o mesmo apresentava alergia ao material da fita e posteriormente foi esclarecido quanto a segurança e a confidencialidade dos dados coletados. E os benefícios apresentados pela pesquisa, visaram minimizar e/ou eliminar a dor lombar dos funcionários, em virtude da correta aplicação da KT, gerando melhora da qualidade de vida e contribuindo para um melhor conhecimento e divulgação desta técnica na sociedade e entre os profissionais fisioterapeutas atuantes nesta área.

\section{RESULTADOS E DISCUSSÕES}

A amostra foi composta por um número $(\mathrm{N})$ de 50 funcionários, a faixa etária mostrou que $22(44 \%)$ dos participantes tinham idade de 18 a 29 anos, 22 (44\%) tinham idade de 30 a 39 anos e 6 (12\%) tinham idade maior ou igual a 40 anos, sendo 18 (36\%) funcionários do sexo masculino e $32(64 \%)$ do sexo feminino, que corresponde ao total da amostra (Tabela 1).

Tabela 1 - Informações gerais dos funcionários avaliados na pesquisa.

\begin{tabular}{|c|c|c|c|c|c|c|c|c|}
\hline & \multicolumn{6}{|c|}{ Grupo } & \multicolumn{2}{|c|}{ Total } \\
\hline & \multicolumn{3}{|c|}{ GKT } & \multicolumn{3}{|c|}{ GC } & & \\
\hline & $\mathbf{n}$ & $\%$ & $P$ & $\mathbf{n}$ & $\%$ & $P$ & $\mathbf{n}$ & $\%$ \\
\hline Faixa etária & & & 0,819 & & & 0,515 & & \\
\hline $18-29$ anos & 13 & 52,0 & & 9 & 36,0 & & 22 & 44,0 \\
\hline $30-39$ anos & 9 & 36,0 & & 13 & 52,0 & & 22 & 44,0 \\
\hline$\geq 40$ anos & 3 & 12,0 & & 3 & 12,0 & & 6 & 12,0 \\
\hline Sexo & & & 0,656 & & & 0,339 & & \\
\hline Masculino & 9 & 36,0 & & 9 & 36,0 & & 18 & 36,0 \\
\hline Feminino & 16 & 64,0 & & 16 & 64,0 & & 32 & 64,0 \\
\hline TOTAL & 25 & 100,0 & & 25 & 100,0 & & 50 & 100,0 \\
\hline
\end{tabular}

Fonte: Lima HC, Luz KRG, Magalhães MM, et al., 2018.

Baseado nos dados contidos no Gráfico 1, pode-se observar que houve redução do quadro álgico no GKT após a aplicação da fita, sendo estatisticamente significante $(p=0,05)$. Enquanto no $G C$ que não foi submetido a nenhum tipo de intervenção, não houve redução significativa da dor $(p=0,001)$. Visto que a dor leve não foi referida por nenhum dos avaliados e após o tratamento obteve-se um percentual de $56 \%$, mostrando assim, que os funcionários que sentiam dor moderada e intensa passaram a sentir dor leve de acordo com a escala visual analógica - EVA, a dor moderada teve uma prevalência de $72 \%$ no início, sendo reduzido para $40 \%$ após a retirada da fita, a dor intensa teve um percentual de $28 \%$ antes e reduziu para $4 \%$ após a aplicação da KT. 
Gráfico 1 - Relação da avaliação da dor pela escala EVA de ambos os grupos.

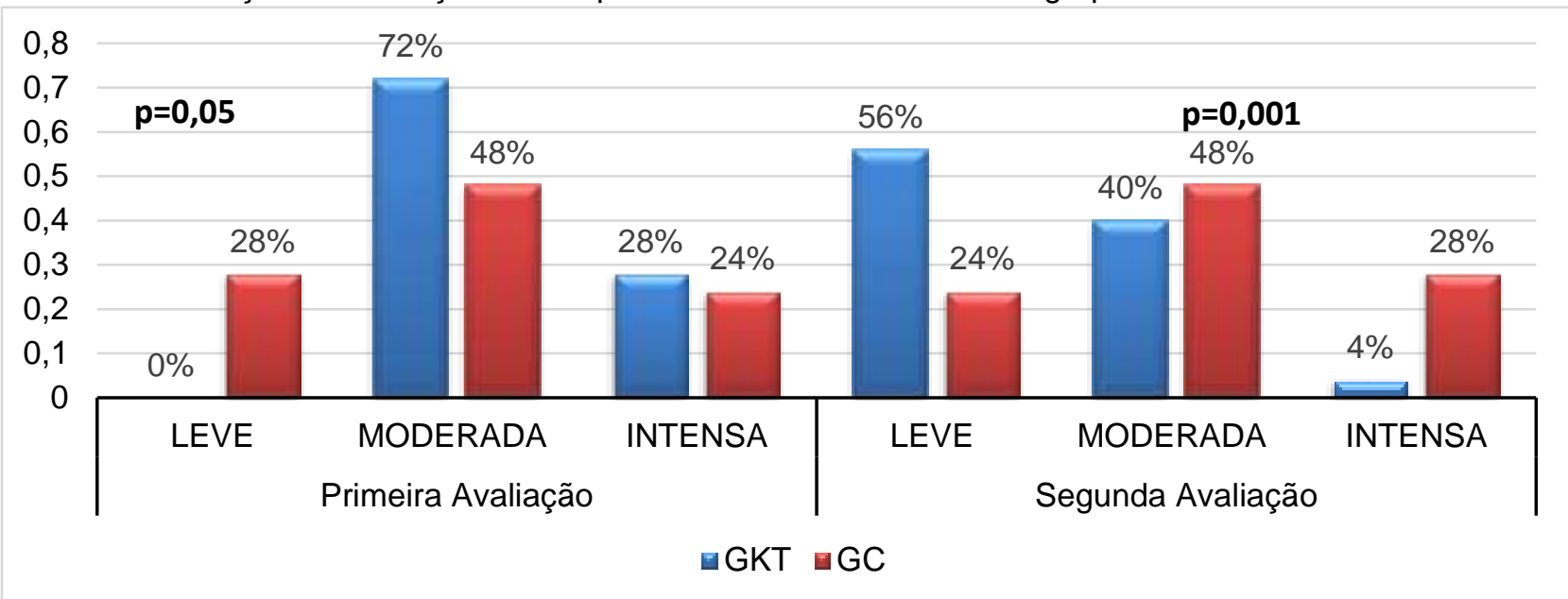

Fonte: Lima HC, Luz KRG, Magalhães MM, et al., 2018. p= teste qui-quadrado.

Um estudo semelhante realizado com sedentários que tinham alteração postural, dor lombar e hérnia de disco afirmou que a utilização da KT por três dias no grupo tratado proporcionou a redução da dor lombar com significância $(p=0,05)$, enquanto que no grupo controle que não foi sujeito a nenhuma intervenção, não houve melhora significativa $(p=0,05)$ segundo a escala EVA (WENKE $R$, et al., 2016).

Outro estudo que fez uso da KT com duração de três dias para a redução da dor lombar em costureiras, também verificou melhora significativa do quadro álgico e da flexibilidade após o tratamento $(p=0,005)$. A tensão provocada pela fita pode inibir os mecanismos da dor e favorecer a analgesia, por meios de estímulos aferentes de grande diâmetro para as fibras nervosas sensitivas, além de promover melhora da circulação sanguínea e linfática e favorecer a propriocepção por meio da excitação dos mecanorreceptores cutâneos (ALONSO AC, et al., 2015).

Os resultados dispostos no gráfico 2 mostram que na comparação da flexão de tronco antes e pós tratamento, houve uma melhora significativa para o GKT após a retirada da fita $(p=0,000)$. Já para o $\mathrm{GC}$ os resultados não foram relevantes ( $p=0,001)$. Uma vez que $56 \%$ dos funcionários mediram de 11 a $20 \mathrm{~cm}$ antes da intervenção e posteriormente esse percentual reduziu para $44 \%$, e aumentou o percentual no escore de 0 a $10 \mathrm{~cm}$ mostrando assim que os funcionários que tinham medidas maior que $10 \mathrm{~cm}$ reduziram para este valor, o que afirma o ganho de mobilidade lombar.

Gráfico 2. Resultado da prevalência da medição mão-terra de ambos os grupos.

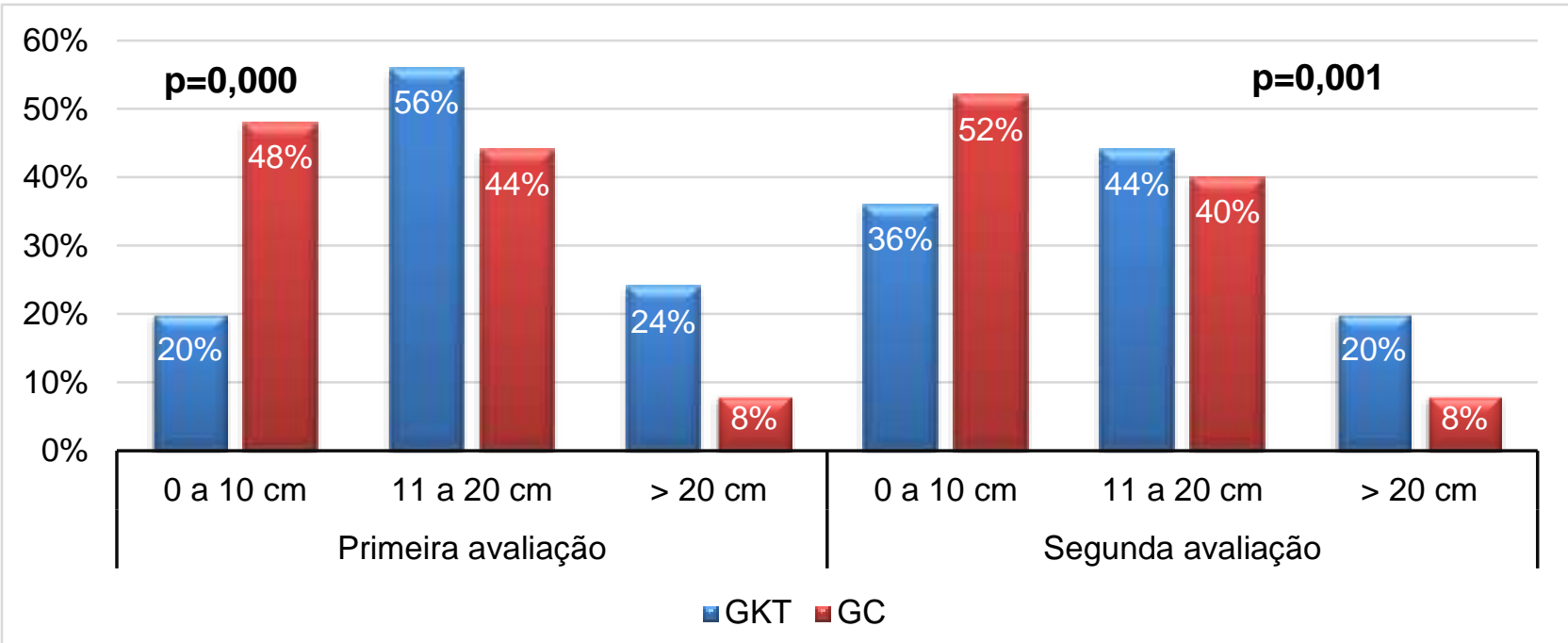

Fonte: Lima HC, Luz KRG, Magalhães MM, et al., 2018. $p=$ teste qui-quadrado. 
Corroborando com dois estudos, um Piloto, que observou uma melhora significativa da dor e da amplitude de movimento na flexão de tronco em pacientes com lombalgia $(p=0,017)$. E um estudo de caso que afirmou que o Método KT foi eficaz nos escores intensidade da dor, atividades de vida diárias e flexibilidade de tronco (ALONSO AC, et al., 2015; ALVES TFM, et al., 2014).

Uma revisão sistemática que abordou os efeitos da KT na síndrome da dor patelofemoral, apontou que os artigos estudados relataram que, além da redução da dor, houve um ganho da flexibilidade em isquiotibiais, afirmando dessa maneira que a utilização da KT de forma correta pode favorecer o ganho de amplitude de movimento (DE OLIVEIRA LMR e GUIMARÃES LAM, 2013).

Contudo é importante ressaltar que houve estudos que visaram avaliar os efeitos da bandagem na mobilidade da coluna lombar e não encontraram resultados significativos, pois observaram melhora tanto no grupo experimental como no grupo controle (ANDRADE J, 2014; DE LIMA EA, et al., 2016).

A mobilidade da coluna lombar é bastante avaliada pelo fato de ser muito importante na realização dos movimentos e pela incapacidade funcional que é gerada quando não há flexibilidade, comprometendo a qualidade de vida os indivíduos. Quando ocorre uma diminuição de amplitude de movimento nessa região com perda de flexão, esta resulta em quadro álgico intenso podendo levar o indivíduo a imobilizar-se (HOPPENFELD S, 2003).

Acredita-se que os resultados positivos gerados pela KT em relação ao acréscimo de flexibilidade, ocorrem pelo fato de que as convoluções que são geradas na pele, elevam os tecidos moles e fáscias, aumentam o espaço da área inflamada e consegue descomprimir os nociceptores, e isso associado ao aumento da irrigação sanguínea e linfática, promove o alívio da dor, que na maioria das vezes está relacionada a diminuição da mobilidade, obtendo assim o aumento da amplitude de movimento (ANDRADE J, 2014).

Os resultados obtidos na avaliação da dor pela Escala Visual Analógica - EVA dispostos no Gráfico 3, demonstram que no GKT 18 (36\%) dos indivíduos referiram dor moderada enquanto no GC 12 (24\%) relatam sentir essa mesma intensidade, ou seja $60 \%$ dos participantes sentiam dor moderada, tornando assim esse nível a mais prevalente.

Gráfico 3 - Resultados da prevalência da avaliação da intensidade da dor pela escala EVA.

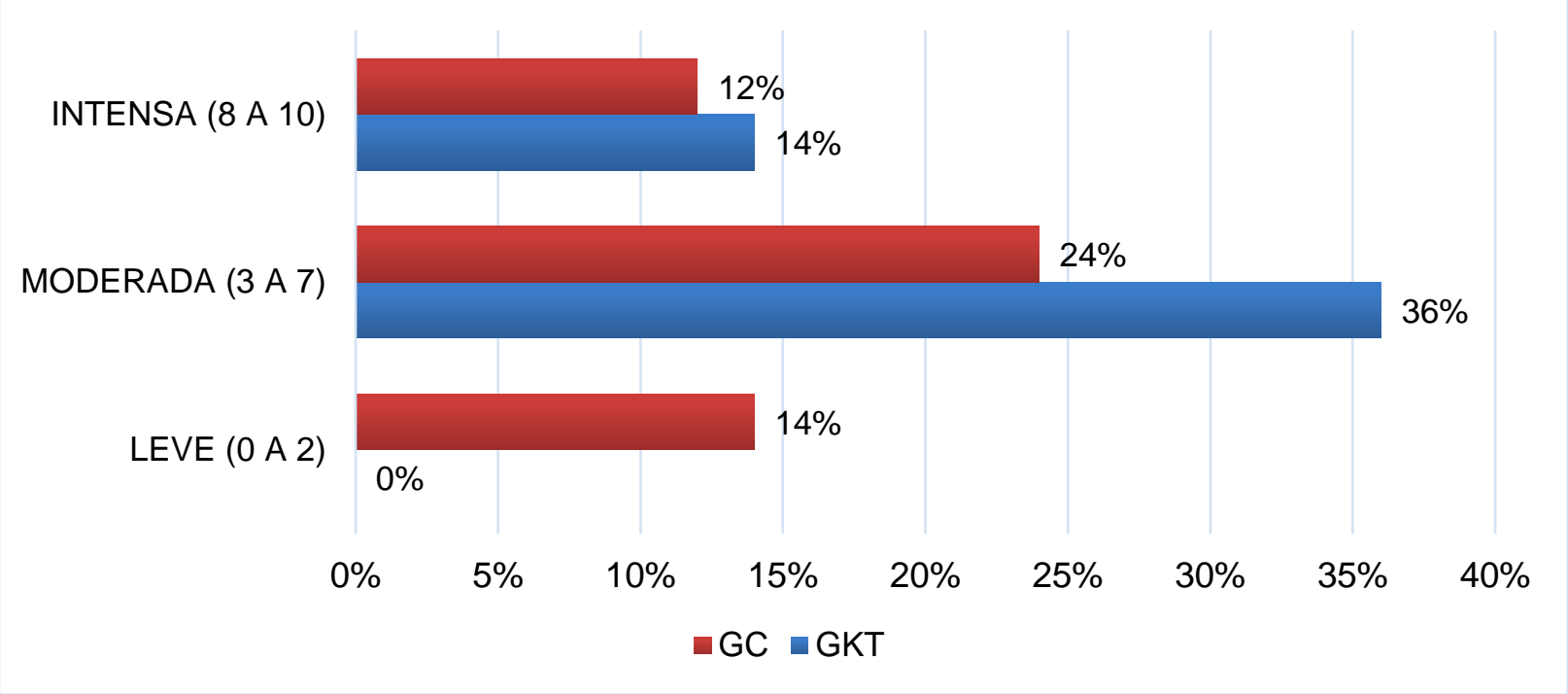

Fonte: Lima HC, Luz KRG, Magalhães MM, et al., 2018.

Jorge EM et al. (2012); realizaram um estudo com trabalhadores que atuavam na posição sentada, na qual utilizaram uma amostra com 21 indivíduos alocados em três grupos, e puderam observar a prevalência de dor moderada, sendo referida por 16 dos colaboradores. Constatou-se em outro estudo sobre lombalgia mecânica, a prevalência de dor lombar moderada no grupo experimental (SALVADOR D, et al., 2011). 
Porém vale ressaltar que esses dados discordam com a maior parte dos estudos encontrados na literatura, visto que a maioria dos artigos comprovaram uma prevalência de dor lombar intensa. Assim fica difícil determinar a etiologia que leva a um quadro de dor mais intensa, contudo é necessário se atentar qual o nível de sofrimento e desconforto pode está mais ligado as características da dor do que exclusivamente com a sua intensidade (MASCARENHAS CHM e SANTOS LS, 2011).

\section{CONCLUSÃO}

Com base nesse estudo pôde-se verificar que o método KT foi eficaz para a redução do quadro álgico na região lombar, sendo estatisticamente significativo com $P=0,05$. Obteve-se também aumento da flexibilidade da coluna lombar, quando comparados os dados da medição mão-terra antes e após a aplicação, sendo extremamente significativo com $p=0,000$. E pôde-se constatar ainda que a maioria dos funcionários avaliados referiram dor de intensidade moderada de acordo com a escala EVA e que apesar dos fundamentos efetivos à aplicação do método na melhora das algias lombares apresentados até o momento, é valido ressaltar que as evidências são restritas, mas as intervenções com a KT mostraram-se eficazes para as alterações lombares. Com isso é importante mencionar que a Kinesio Taping atua na percepção da dor dos indivíduos, mas a intervenção exerce pouco efeito sobre a causa base, portanto essa modalidade deve ser agregada ao rol de técnicas da fisioterapia, devendo sempre que possível ser associada a outras terapias que visem tratar a origem da dor.

\section{REFERÊNCIAS}

1. ALONSO AC, et al. O efeito do uso da bandagem funcional no tratamento da dor lombar em costureiras, estudo piloto. Revista CPAQV-Centro de Pesquisas Avançadas em Qualidade de Vida, v. 7, n. 1, 2015.

2. ALVES TFM, et al. Efeitos do método kinesio taping em costureiros com dor lombar inespecífica. Revista De Trabalhos Acadêmicos-Universo Recife, v. 1, n. 1, 2014.

3. ANDRADE J. Os efeitos da Banda Neuromuscular na mobilidade da coluna lombar. 2014. Trabalho de Conclusão de Curso. Natal. Universidade Federal do Rio Grande do Norte Centro de Ciências da Saúde. Departamento De Fisioterapia.

4. BARROS SS, et al. Lombalgia ocupacional e a postura sentada. Rev. dor, São Paulo, v. 12, n. 3, p. 226230, Sept. 2011.

5. CARAVIELLO EZ, et al. Avaliação da dor e função de pacientes com lombalgia tratados com um programa de Escola de Coluna. Rev Acta fisiátrica, v. 12, n. 1, p. 11-14, 2016.

6. DE LIMA EA, et al. Avaliação do nível álgico antes e após intervenção fisioterapêutica na lombalgia aguda (Relato De Caso). Revista Interfaces: Saúde, Humanas e Tecnologia, v. 2, n. 6, 2016.

7. DE OLIVEIRA LMR; GUIMARÃES LAM. Bandagem funcional na sindrome da dor patelofemoral: Uma revisão sistemática. 2013.

8. DE OLIVEIRA LR; MEJIA DPM. O efeito da bandagem funcional elástica na dor lombar. 2012.

9. FREITAS KPN, et al. Lombalgia ocupacional e a postura sentada: efeitos da cinesioterapia laboral. Revista Dor, v. 12, n. 4, p. 308-13, 2011.

10. HOPPENFELD S. Propedêutica ortopédica: coluna e extremidades. São Paulo: Atheneu, 2003.

11. JORGE EM, et al. Kinesiology Taping nas lombalgias de trabalhadores que atuam na posição sentada. Revista Trances, v. 4, n. 3, p. 181-206, 2012.

12. LIMA JMR. Aplicação da banda neuromuscular e seus efeitos na alteração da flexibilidade da coluna lombar. 2016. Trabalho de Conclusão de Curso. Universidade Federal do Rio Grande do Norte. Departamento de Fisioterapia.

13. MASCARENHAS CHM, SANTOS LS. Avaliação da dor e da capacidade funcional em indivíduos com lombalgia crônica. J Health Sci Inst, v. 29, n. 3, p. 205-8, 2011.

14. PATARO SMS, FERNANDES RCP. Heavy physical work and low back pain: the reality in urban cleaning. Rev. bras. epidemiol., São Paulo, v. 17, n. 1, p. 17-30, Mar. 2014.

15. REINEHR FB, et al. Influência do treinamento de estabilização central sobre a dor e estabilidade lombar. Revista Fisioterapia em Movimento, v. 21, n. 1, 2017.

16. SALVADOR D, et al. Aplicação de técnica de energia muscular em coletores de lixo com lombalgia mecânica aguda. Fisioterapia e Pesquisa, v. 12, n. 2, p. 20-27, 2011.

17. THOMPSON D. Bandagem funcional-Aspectos teóricos. Revista Terapia Manual [online]. 2010.

18. VARGAS B, et al. Uso da kinesio taping na dor lombar: revisão sistemática. Revista ConScientiae Saúde, v. 13, n. 1, 2014.

19. WENKE R, et al. Efeitos da aplicação da bandagem elástica em sedentários com alteração postural, Hipermobilidade Lombar e Hérnia de disco. Revista Uniandrade, v. 17, n. 3, p. 114-120, 2016. 